\title{
ANÁLISIS DEL PROCESO PRODUCTIVO DE UNA EMPRESA DE CONFECCIONES: MODELACIÓN Y SIMULACIÓN
}

\section{A DRESSMAKING FACTORY PRODUCTION PROCESS ANALYSIS: MODELING AND SIMULATION}

Paola A. Sánchez', Fernando Ceballos², Germán Sánchez Torres ${ }^{3}$

Fecha de recepción: 24 de Septiembre de 2014

Fecha de aprobación: 27 de Julio de 2014

Referencia: P.A. Sánchez, F. Ceballos, G. Sánchez Torres. (2015). Análisis del proceso productive de una empresa de confecciones: modelación y simulación. Ciencia e Ingeniería Neogranadina, 25 (2), pp 137 - 150, DOl: http://dx.doi. org/10.18359/rcin.1436

\section{RESUMEN}

El modelado y simulación de pequeños sistemas industriales ha adquirido gran importancia en los procesos de toma de decisiones, toda vez que les permite a microempresas fundamentar sus decisiones de producción. Este artículo hace un análisis de una empresa dedicada a la confección de ropa exterior masculina con una producción semanal promedio de 490 prendas. La motivación del estudio radica en el interés de incrementar su productividad; por esto, se desarrolló un modelo del sistema real con la herramienta Arena ${ }^{\circledR}$, logrando identificar las partes de los procesos responsables de atrasos y "cuellos de botella" que permitieron plantear el escenario de solución. Este documento muestra los resultados de la simulación del sistema real y una comparación con un escenario de mejora que presentó un aumento en la productividad.

Palabras clave: Simulación Discreta, Modelamiento, Confecciones, Toma de Decisiones, Arena ${ }^{\circledR}$.

1. Ingeniera Industrial, Doctora en Ingeniería de Sistemas, docente investigador, Universidad Simón Bolívar, Barranquilla, Colombia, psanchez9@ unisimonbolivar.edu.co

2. Ingeniero de Sistemas, Doctor en Ingeniería de Sistemas, profesor asistente, Universidad de Antioquia, Medellín, Colombia, yony.ceballos@udea. edu.co

3. Ingeniero de Sistemas, Doctor en ingeniería de sistemas, profesor asistente, Universidad del Magdalena, Santa Marta, Colombia, gsanchez@ unimagdalena.edu.co 


\section{ABSTRACT}

Modeling and simulation of small industrial systems, has become important in the decisionmaking processes, because it allows small companies to make their production decisions. This article analyses a company engaged in the manufacture of men's outwear with average weekly production of 490 units. The motivation of this study lies in the interest the company owners have to increase its productivity; for this reason, a model of the real system with Arena ${ }^{\circledR}$ tool was developed. With this model, it was possible to recognize the process parts that cause delays and bottlenecks and from there it was suggested a valid solution scenario. This paper shows the results of real system simulation and proposes two scenarios which provide an increase in the productivity.

Keywords: Discrete Simulation; Modeling; Dressmaking factory; Decision Making; ARENA ${ }^{\circledR}$.

\section{INTRODUCCIÓN}

En la actualidad, en el sector empresarial existe la necesidad de ser cada día más competitivos, lo que obliga a las organizaciones a analizar sus procesos para obtener una mejor calidad que le permita cumplir con las necesidades y expectativas de los clientes. La competitividad empresarial, en el contexto de la globalización, exige a las organizaciones para ser sostenibles en mercados nacionales e internacionales tener una administración de los procesos productivos más eficiente y eficaz de sus recursos financieros, humanos, tecnológicos, entre otros [1].

La toma de decisiones es un proceso que puede generar diferentes impactos en los procesos productivos. Dichas decisiones se pueden realizar empleando técnicas de investigación de operaciones en función del nivel de complejidad de los problemas, del costo que acarrea dicha decisión y de la información conocida al momento de tomar la decisión. Por tanto, en las pequeñas y medianas empresas utilizan técnicas de toma de decisiones basadas en la experiencia de los actores de los procesos o con experiencias exitosas en otras empresas, pero sin la posibilidad de validar la eficiencia de las decisiones [2-3]. Es necesario que estas empresas cuenten con unas herramientas de toma de decisiones que no representen una inversión significativa y que permitan hacer ensayos de las configuraciones ideales analizadas en el proceso [4-5], como la simulación computacional. Los modelos de análisis y simulación son llevados a cabo con el objetivo de conocer mejor sistemas complejos, para desarrollar y probar nuevos recursos, procesos, políticas o sistemas con las expectativas de fabricación modernas sin la necesidad de desarrollarlos en la realidad, reuniendo información y conocimiento sin perturbar el sistema actual [6].

La simulación de sistemas es una alternativa para conocer de forma acertada los puntos 
críticos que pueden tener los procesos de producción de una empresa y con estos modelar soluciones que incrementen la eficiencia y que reduzcan los tiempos en las diferentes actividades realizadas durante la producción de un artículo o la prestación de un servicio.

Este artículo hace un análisis de los procesos dentro de la cadena de producción de una empresa dedicada a la confección de ropa exterior masculina del sector de confecciones. La confección es entendida como la transformación de un conjunto de partes o piezas hechas de textiles en prendas de vestir. En Colombia, esta industria es considerada madura y en crecimiento, desarrollada en todo el país con focos principales en Bogotá, Medellín y Cali, y representa el 1,17 \% del PIB nacional y el 9,82\% de la industria manufacturera [7].

Diferentes trabajos se han realizado con el objetivo de integrar estrategias computacionales que permitan analizar y optimizar los procesos de producción en la industria de confecciones. En orden cronológico, en [8] se muestra la aplicación de la simulación para optimizar las variables del proceso de planificación de la producción como la disponibilidad de materiales, la relación entre la eficiencia del sistema y las órdenes de producción en relación con las preferencias de los clientes en color del producto. En [9] se analizaron los efectos, en el diseño de instalaciones de producción, de diversos factores como disposición del producto, tiempos de inactividad de las máquinas, tamaños de los lotes y la capacidad de transporte de productos. Esto les permitió concluir que considerar varios parámetros en conjunto al diseñar las instalaciones podría evitar el deterioro del rendimiento. En [10] se propuso un modelo general para el proceso de producción en empresa textil utilizando dinámica de sistemas cuyo objetivo era identificar los elementos que se deben integrar a los costos de producción en las pequeñas y medianas empresas de confección. Este análisis permitió la construcción de un software para modelar diferentes escenarios con el objetivo de optimizar el proceso. En [11] se utilizaron modelos de simulación de eventos discretos para modelar el departamento de costura de una compañía de confecciones, lo que permitió estimar los efectos de diferentes configuraciones en tiempo y capacidad de producción, eliminación de retardos y optimización de recursos. En [12] se describe una metodología para mejorar el sistema de producción en pequeñas y medianas empresas y se modela el sistema mediante eventos discretos. El objetivo fue evaluar diferentes escenarios que incluyen las llamadas prioridades competitivas de las cuales la calidad y el tiempo de entrega resultaron ser las más relevantes. Un trabajo similar en la industria textil es [13] en el cual se busca optimizar los horarios de trabajo en una línea múltiple de confección de prendas de vestir.

Sin importar la técnica de simulación utilizada, la literatura demuestra que esta constituye una herramienta fundamental para optimizar los procesos productivos de las empresas.

El objetivo del estudio se centró en el análisis del proceso productivo de una empresa nacional para incrementar la productividad identificando las partes de los procesos responsables de atrasos y "cuellos de botella". La empresa analizada está caracterizada en la franja de pequeñas y medianas empresas con tres años de trayectoria dedicada fundamentalmente a la confección de ropa exterior masculina. Aunque la empresa elabora diferentes prendas, el análisis se concentra en el proceso 
de producción de camisas debido a que esta constituye el producto de mayor demanda y con la mayor participación en los ingresos de la compañía. El aporte principal consiste en mostrar al sector, de forma específica representada en un caso de aplicación real, las bondades de la incorporación de herramientas tecnológicas que le permiten mejorar sus procesos.

\section{MATERIALES Y MÉTODOS}

La metodología utilizada para analizar el proceso de producción de la empresa de confecciones se describe en la figura 1.

Específicamente, el esquema metodológico utilizado posee tres etapas: formulación del problema, desarrollo del modelo y experimentos de simulación. En la formulación del problema se atiende y define la necesidad particular de la empresa de estudio, así como la recolección de los datos iniciales relacionados con el proceso de producción. En el desarrollo del modelo el objetivo principal es la descripción y comprensión del modelo actual que opera, lo que permitirá construir el modelo computacional y junto con los datos recolectados validar la formulación obtenida. Finalmente, el modelo resultante es utilizado para proponer nuevos escenarios de solución y analizar el resultado de la incorporación dentro del sistema actual.

Para el proceso de validación se requiere emplear una metodología que permita tener en cuenta las características propias de sistema modelado, este trabajo adoptó la metodología de validación descrita en la figura 2.

\section{RESULTADOS Y DISCUSIÓN}

\subsection{FORMULACIÓN DEL PROBLEMA}

El nivel de ingresos percibido por la empresa correspondiente a la producción de camisas, no está dentro de los valores típicos de empresas del mismo sector, en situaciones comparables en relación con el número de máquinas y operarios. Lo anterior evidencia la necesidad de hacer un análisis del proceso productivo [15-16], mediante alguna técnica formal como la simulación [17-18]. En el caso de las empresas de confecciones, se puede

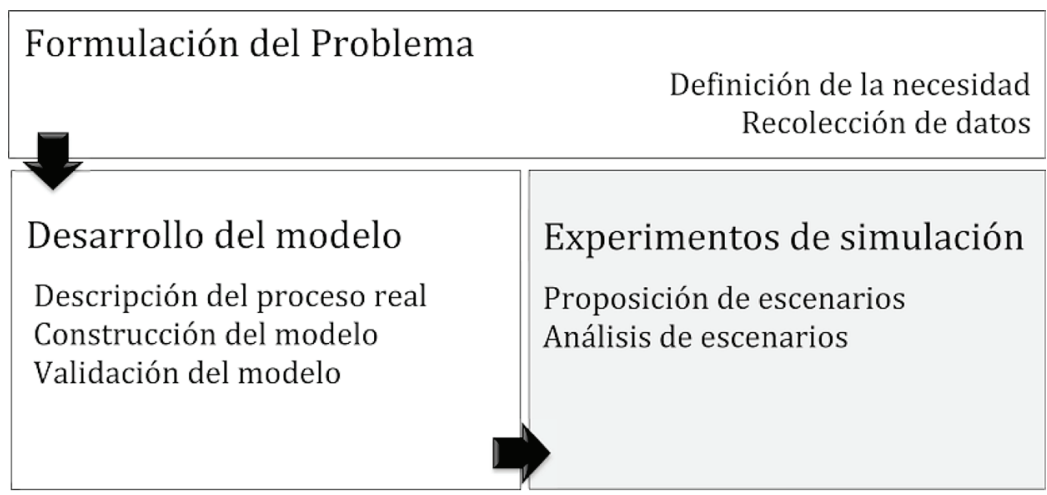

Figura 1. Diagrama de la metodología utilizada.

Fuente: elaboración propia. 


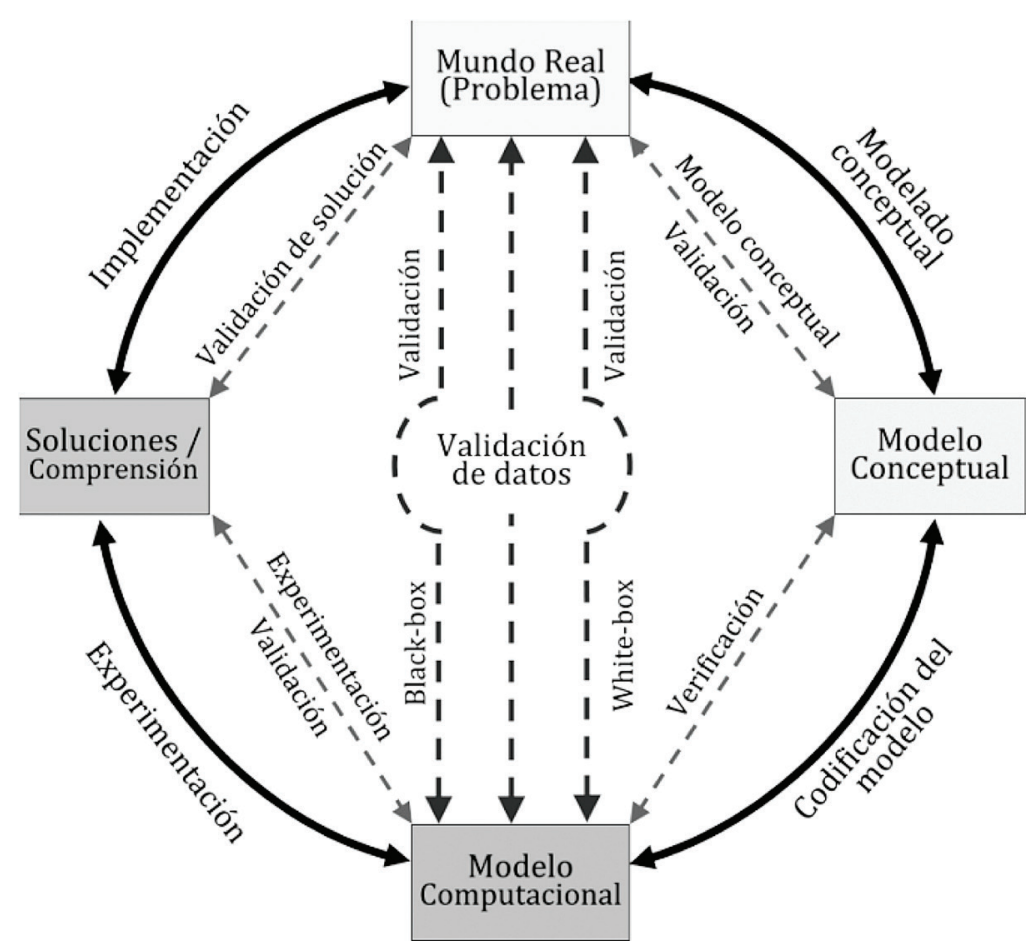

Figura 2. Proceso de validación de modelos (Modificado de [14]).

Fuente: Elaboración propia.

emplear la simulación para caracterizar las etapas del proceso productivo en conjunto con las estaciones de trabajo involucradas. Especialmente las estaciones asociadas con operaciones manuales como son: máquinas planas, fileteadoras, ojaladoras y en menor medida las máquinas botonadoras. En dichas máquinas, la necesidad de tener un operario disponible para que haga control del acceso de la prenda al proceso puede generar demoras por diferentes problemas, tales como un atascamiento del material, averías en la máquina y nivel de entrenamiento del operario.

Se obtuvieron datos reales tomados en múltiples días de trabajo de la empresa. La muestra se tomó en una semana durante 8 horas diarias en cada una de los puestos de servicio del proceso productivo.

\subsection{DESARROLLO DEL MODELO}

El proceso de simulación se realiza tomando como insumo la estructura real del proceso productivo.

\section{Descripción del proceso real}

El proceso real del taller produce 80 unidades diarias de camisas, para un total aproximado de 490 unidades semanales, con una intensidad horaria laboral de 48 horas por semana. Cuenta con 16 máquinas planas, una fileteadora puntada de seguridad, una ojaladora, una botonadora, dos planchas industriales y una plana dos agujas, siendo en total 22 máquinas. La empresa tiene contratados 26 empleados, distribuidos de la siguiente manera: uno para 
cada máquina, dos al manejo de las planchas y cuatro más para la terminación y empaque del producto.

En el proceso las partes cortadas que forman una camisa se entregan al confeccionista, los bolsillos, los cuellos, las perillas, los delanteros, las tapas, las almillas, las espaldas y las mangas. Detalladamente, al comienzo pueden llevarse a cabo varios procesos en paralelo: en las planchas se prehorman los bolsillos y se pega la entretela a los cuellos y a las perillas; en una máquina plana son preparadas las tapas y en la fileteadora se unen las almillas a las espaldas. Luego los bolsillos y los cuellos con la entretela pasan por máquinas planas para dejarlos preparados; igualmente, los delanteros de las camisas y las perillas con la entretela pasan por la máquina plana dos agujas para dejar finalizados los delanteros con las perillas. Para continuar con el proceso de producción, los bolsillos preparados se pegan en los delanteros y luego se pegan las tapas, ambos procesos utilizan máquinas planas. El resultando de este proceso son los delanteros con los bolsillos completos.

Posteriormente, se deben pegar los frentes con las espaldas utilizando la fileteadora para obtener las camisas con el frente y la espalda ya listas. Cuando las camisas están listas con las mangas se cierran en la fileteadora, se les hace el ruedo con una de las máquinas planas, se hacen los ojales con la ojaladora y se les ponen los botones con la botonadora. En este punto del proceso de producción las camisas están casi terminadas, las operaciones faltantes la constituyen la primera parte de la terminación, que consta de pulir las hebras sobrantes y revisar la calidad de la prenda, luego pasa a ser planchada y, por último, nuevamente, a proceso de terminación donde se doblan y empacan. En general, el proceso es simple, debido a que la secuencia es un estándar en el proceso de confección de la empresa.

En la figura 3 se presenta detalladamente el flujo de una prenda en todo el proceso productivo.

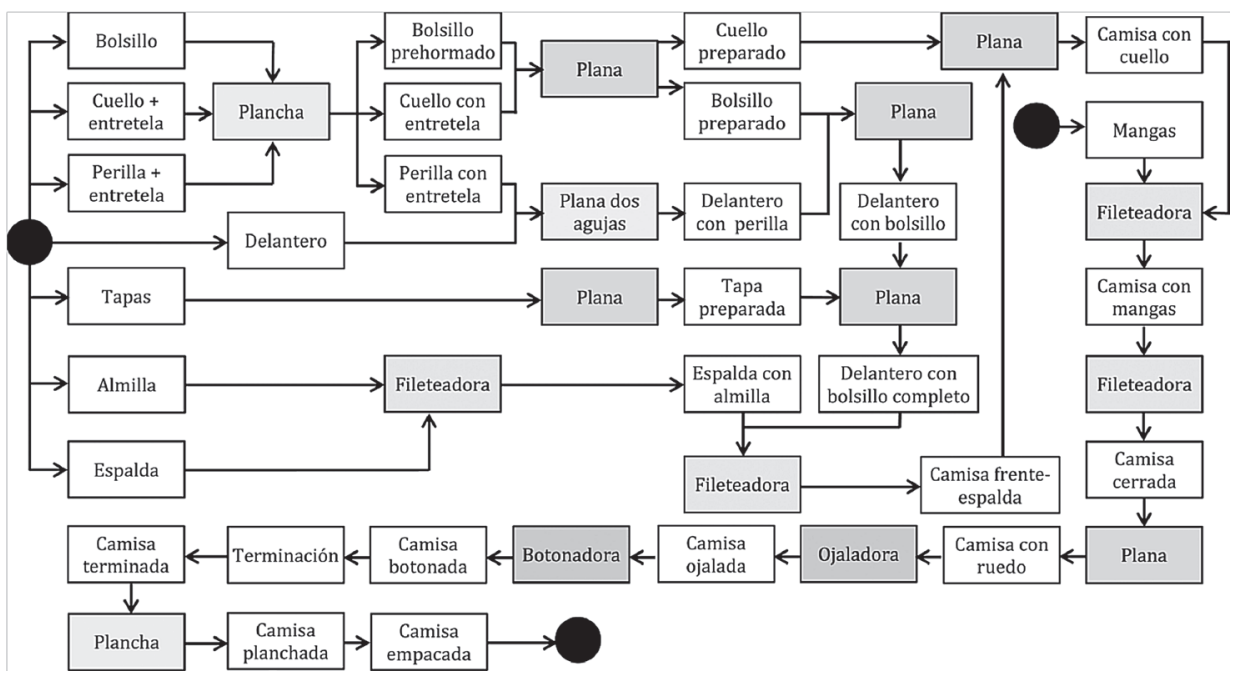

Figura 3. Diagrama actual de proceso de confección de camisas.

Fuente: Elaboración propia. 
El proceso inicia cuando se tiene en el gráfico un círculo oscuro al extremo izquierdo, del cual se pueden realizar múltiples actividades y finaliza con otro círculo oscuro que solo posee flechas de entrada.

La construcción de un diagrama de procesos permitió obtener un modelo inicial desde una secuencia de ensamble lógica. Posteriormente, este modelo lógico fue implementado empleando un software de uso específico para simulación de eventos discretos. El sistema de la empresa de confecciones se simuló con la herramienta Arena ${ }^{\circledR}$ en su versión 14.0 [19]. Todos los procesos para la confección de una camisa fueron simulados y concatenados hasta obtener el producto final durante una hora.

\subsubsection{Construcción del modelo}

La implementación se puede observar en figura 4. La figura 4a ilustra el esquema general,

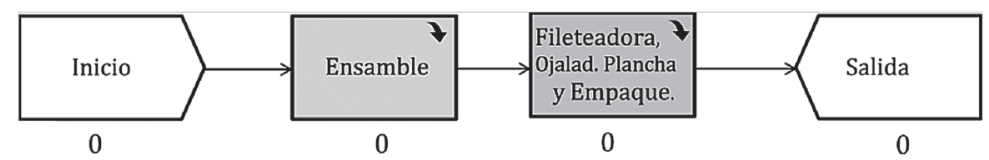

a)

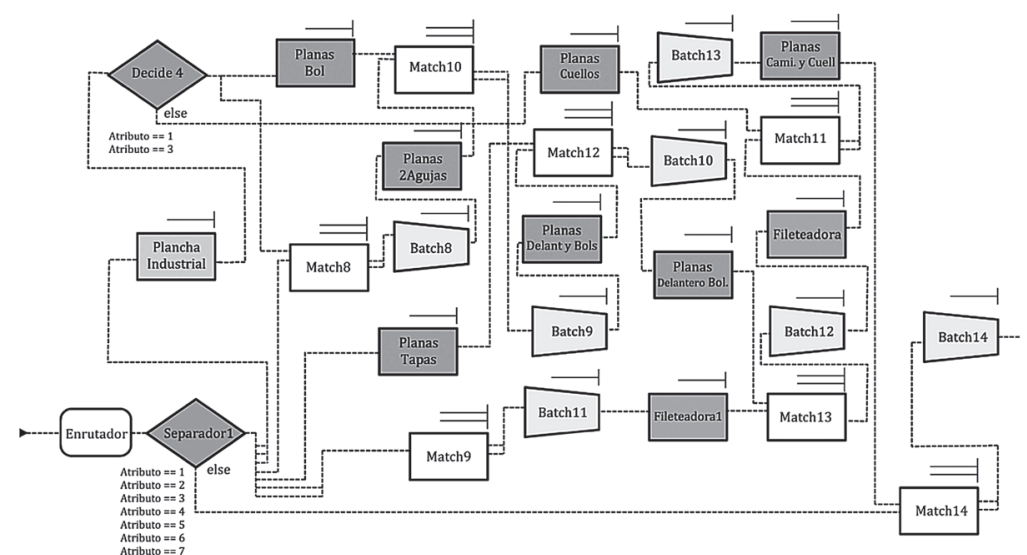

b)
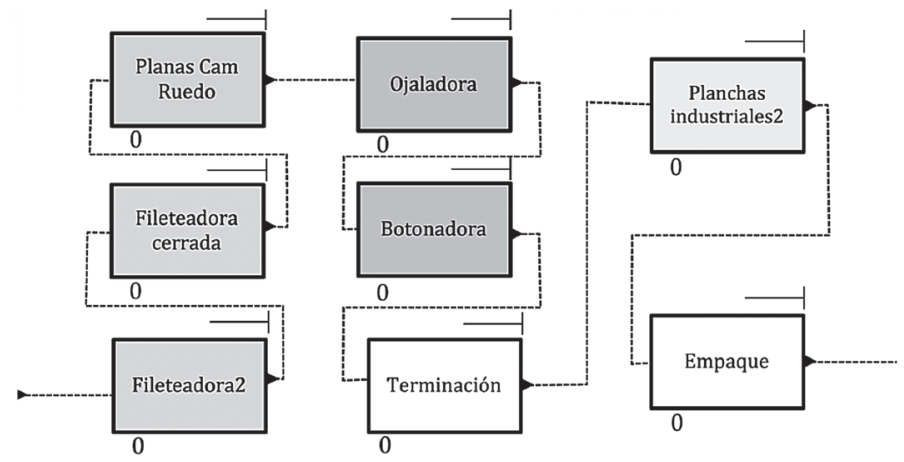

c)

Figura 4. Representación en ARENA® del proceso actual de confección de camisas, a) esquema general, b) sub modelo de ensamble y c) sub modelo ojaladora-plancha-empaque.

Fuente: Elaboración propia. 
los sub modelos de ensamble y el modelo ojaladora-plancha-empaque se muestran en la figura $4 b$ y $4 c$, respectivamente. Para representar el conjunto de máquinas se emplea dentro de las entidades el objeto SET, el cual permite agrupar la disponibilidad de las mismas en los objetos presentados y utilizar los recursos de la forma en la cual se requieran. Para una simulación correcta en el software se insertaron varios elementos etiquetadores que nombran cada parte de la camisa que llega a la empresa, es decir, los bolsillos, los cuellos, las perillas, los delanteros, las tapas, las almillas, las espaldas y las mangas.

Inicialmente, se requiere conocer las distribuciones de llegadas a cada una de las entidades identificadas en el sistema; en la tabla 1 se presenta un consolidado de tal información. Todas las distribuciones se

Tabla 1. Distribuciones Estadísticas asociadas a cada proceso del sistema.

\begin{tabular}{|c|c|c|}
\hline Entidades & Proceso & Distribución (segundos) \\
\hline \multirow{3}{*}{ Planchas Industriales } & Bolsillos & \multirow{3}{*}{$N\left(\mu=54,12 ; \sigma^{2}=3,08\right)$} \\
\hline & Cuellos & \\
\hline & Perillas & \\
\hline \multirow{8}{*}{ Planas } & Camisa Terminada & $\log N\left(\mu=180,15 ; \sigma^{2}=3,23\right)$ \\
\hline & Bolsillos & $N\left(\mu=48,96 ; \sigma^{2}=2,91\right)$ \\
\hline & Cuellos & $U(a=70 ; b=80)$ \\
\hline & Tapas & $N\left(\mu=54,14 ; \sigma^{2}=2,38\right)$ \\
\hline & Bolsillo/Delantero & $U(a=103 ; b=113)$ \\
\hline & Bolsillo-Delantero/Tapa & $N\left(\mu=55,21 ; \sigma^{2}=2,45\right)$ \\
\hline & Camisa-Frente-Espalda/Cuello & $U(a=45 ; b=55)$ \\
\hline & Ruedo & $N\left(\mu=28,21 ; \sigma^{2}=2,12\right)$ \\
\hline \multirow{5}{*}{$\begin{array}{c}\text { Plana dos agujas } \\
\text { Fileteadora }\end{array}$} & Perilla & $N\left(\mu=118,5 ; \sigma^{2}=4,38\right)$ \\
\hline & Almillas-Espaldas & \multirow{4}{*}{$N\left(\mu=51,93 ; \sigma^{2}=2,92\right)$} \\
\hline & Delantero-Espalda & \\
\hline & Cerrar Camisa & \\
\hline & Pegar Mangas & \\
\hline Ojaladora & Ojales & $N\left(\mu=89,57 ; \sigma^{2}=3,06\right)$ \\
\hline Botonadora & Botones & $N\left(\mu=89,93 ; \sigma^{2}=3,02\right)$ \\
\hline Terminación inicial & Camisa Terminada & $U(a=115 ; b=125)$ \\
\hline Terminación final & & $N\left(\mu=120,21 ; \sigma^{2}=3,87\right)$ \\
\hline
\end{tabular}

Fuente: Elaboración propia. 
hallaron utilizando el software Statgraphics ${ }^{\circledR}$ XVI [20], mediante un proceso de pruebas de bondad de ajuste aplicado sobre los datos de la muestra obtenida en la empresa. Se emplearon las distribuciones que mayor precisión obtuvieron con respecto a los datos de cada proceso y que fueran aptas para utilizar dentro de los modelos creados en el software Arena ${ }^{\circledR}$.

\subsubsection{Validación del modelo}

Los modelos de simulación generalmente no son instrumentos de proyección con alta precisión, esto se debe al método de integración que generalmente es una técnica de aproximación para la estimación de muestras, el ajuste de las distribuciones $y$ al error en la toma de datos [21-23]. No obstante, es necesario validar y verificar el modelo en relación con su ajuste.

\subsection{EXPERIMENTO DE SIMULACIÓN}

Una vez modelado el sistema y construida su implementación se procedió a evaluar un escenario de solución. En las secciones siguientes se describe el escenario de solución y se muestran los resultados obtenidos.

\subsubsection{Estado actual}

En la tabla 2 se muestran los resultados de la simulación del estado actual del sistema. La simulación es realizada durante un periodo de tiempo de una hora para poder verificar que sea congruente con el sistema real. El número de unidades producidas en el sistema real es 10 unidades, el cual es cercano al modelo presentado que genera 9 unidades.

Tal como se observa en la tabla 2, el nivel de utilización de la máquina plana de dos agujas, fileteadora y las planchas sobrepasa el $35 \%$. Esto indica que no se pueden incrementar las unidades a procesar en la botonadora y la ojaladora, que se alimentan de los productos generados en las máquinas anteriores, lo que evidencia una menor utilización de las mismas.

Tabla 2. Resultados de la simulación del estado actual del sistema.

\begin{tabular}{|c|c|c|}
\hline & Trabajos Completados & Porcentaje de uso \\
\hline Botonadora & 9 & $22,4 \%$ \\
\hline Ojaladora & 9 & $22,4 \%$ \\
\hline Plana dos agujas & 11 & $35,9 \%$ \\
\hline Fileteadora & 39 & $55.9 \%$ \\
\hline Plancha 1 & 32 & $65,3 \%$ \\
\hline Plancha 2 & 32 & $60,8 \%$ \\
\hline \multicolumn{2}{|c|}{ Tiempo promedio en el sistema } & $43.2 \mathrm{~min}$ \\
\hline \multicolumn{2}{|c|}{ Número de trabajos terminados } \\
\hline
\end{tabular}

Fuente: Elaboración propia. 
El número de unidades procesadas es mayor en la fileteadora y las planchas; sin embargo, las planchas realizan una mayor cantidad de procesos, por tanto, para aumentar la productividad se debe intervenir en este recurso. Otra situación importante dentro del modelo es que la botonadora y la ojaladora se pueden emplear para otros procesos de la empresa, ya que cada prenda elaborada demanda un uso escaso de este recurso, lo cual puede orientar la solución de este escenario hacia la diversificación de producción e incurrir en otros mercados.

Después de un acercamiento inicial al sistema y de un reconocimiento primario de sus componentes y características principales, se propone el escenario de solución alternativo que permita incrementar la productividad de la compañía. La evaluación del estado actual de la compañía permitió identificar que existe una estación que genera retrasos en el sistema general y que requiere la ejecución de cantidades considerables de unidades, como las estaciones planchas. Tal como lo proponen algunos autores [24], una alternativa de solución posible es incrementar el número de recursos que producen retrasos.

\subsubsection{Escenario solución: incremento de recurso planchas industriales}

Se propone un aumento en el número de máquinas que podrían estar actuando como un "cuello de botella" en el sistema. Analizando las filas de mayor porcentaje de utilización y aquellas máquinas que más procesos manejaban se simuló el sistema añadiendo una plancha encargada únicamente de recibir las camisas terminadas, liberando este proceso de las otras dos planchas existentes. La solución planteada es viable, toda vez que permite una mejor utilización de las planchas lo que permite una mayor disponibilidad de productos en procesos posteriores.

En la tabla 3 se presentan los resultados de incluir una plancha especializada en el proceso de camisa terminada. Se puede analizar como el total de productos terminados en una hora aumentó en promedio a 10 camisas y el tiempo promedio en el sistema tuvo una variación considerable, al disminuir en un minuto aproximadamente en la elaboración de un producto, con lo que se logró incrementar la productividad de la empresa. Al aumentar el recurso planchas industriales se generó un incremento del $11,11 \%$ en la producción de la semana. Sin embargo, los procesos de planchado deben realizarse en forma paralela, lo cual genera tiempos de ocio en las planchas; a pesar de esto, hay una mejora en la eficiencia por el incremento en el número de trabajos completados, así como en el porcentaje de uso de todas las máquinas, lo que evidencia una mejor utilización de los recursos. En otros estudios, como en [25], se ha identificado que la pérdida en ventajas competitivas y comparativas se fundamenta en factores coyunturales asociados con la remuneración y la creciente mejora tecnológica, lo que ha permitido competir con precio y disminuir considerablemente el impacto generado con la modificación del proceso de manufactura.

\subsection{ANÁLISIS COMPARATIVO}

En el sistema real, la empresa produce 80 unidades diarias de camisas para un total aproximado de 490 unidades semanales con una intensidad horaria laboral de 48 horas por semana. El modelo construido del sistema actual permite replicar de manera aproximada la situación real, donde aproximadamente se 
Tabla 3. Resultados de la implementación del escenario de solución propuesto: Incremento de recurso Planchas Industriales.

\begin{tabular}{|c|c|c|}
\hline & Trabajos Completados & Porcentaje de uso \\
\hline Botonadora & 10 & $24,9 \%$ \\
\hline Ojaladora & 10 & $24,6 \%$ \\
\hline Plana dos agujas & 16 & $50,9 \%$ \\
\hline Fileteadora & 44 & $63,6 \%$ \\
\hline Plancha 1 & 22 & $39,5 \%$ \\
\hline Plancha 2 & 24 & $50,1 \%$ \\
\hline Plancha 3 & 22 & $46,9 \%$ \\
\hline $\begin{array}{c}\text { Tiempo promedio en el } \\
\text { sistema }\end{array}$ & 42,4min \\
\hline $\begin{array}{c}\text { Número de trabajos } \\
\text { terminados }\end{array}$ & 10 \\
\hline
\end{tabular}

producen 72 unidades diarias, que equivaldrían a 432 unidades semanales. El escenario de solución propuesto presenta mejoras en la productividad de la empresa, ya que se aprecia un aumento de una camisa en promedio en una hora, lo cual conduce a un incremento de productividad de un $11,1 \%$, es decir 480 camisas por semana.

Una ganancia notable se ve reflejada en el tiempo promedio de elaboración de una prenda que pasa de 43,2 minutos en el modelo del sistema actual a 42,4 minutos en el escenario propuesto. Estos resultados son congruentes, ya que se está aumentando el número de máquina con mayor retraso de la producción debido a que realizan operaciones fundamentales de la prenda. En el estado actual del sistema solo se tiene una máquina de cada tipo, lo que no es suficiente por lo que aumenta los tiempos en las diferentes colas.
En la literatura se han reportado resultados similares [26]; sin embargo, los contextos de aplicación difieren considerablemente porque cada país aplica políticas laborales distintas.

Otra mejora evidente en la solución propuesta es que se incrementan los porcentajes de uso de las máquinas: botonadora, ojaladora, plana de dos agujas y fileteadora; la nueva distribución hace que se aprovechen de mejor manera los recursos disponibles y se disminuyan los tiempos de ocio de algunas máquinas. En las planchas industriales los porcentajes de ocupación se distribuyen en función de los operarios disponibles. En esta dirección, en estudios similares [27], se han realizado diversos análisis modificando el flujo de materiales y la distribución en planta con lo que se han logrado resultados satisfactorios; sin embargo, es necesario anotar que los resultados difieren en cuanto a 
las características de la compañía y el objetivo que busca el decisor.

Los resultados se deben comparar con el gasto para la adquisición de las nuevas máquinas, lo que constituye inversión económica importante. Sin embargo, se puede considerar que la inversión para la nueva máquina sería recuperable en el tiempo, pues se lograría un aumento en la producción, el cual probablemente impacte positivamente en las ganancias y genere así una estabilidad en la empresa y una confiabilidad para reponer el gasto realizado. En relación con la demanda, la empresa es una maquiladora de una marca ampliamente reconocida, la cual compra la producción realizada. Según información de expertos en la empresa, existen en la zona solo 3 empresas de una capacidad similar que realizan el mismo tipo de productos.

Los recursos denominados operarios pueden mejorar la eficiencia del proceso si estandarizan y alcanzan tiempos nominales de colocación y retiro de la prenda en las máquinas, lo cual incrementaría considerablemente la eficiencia del proceso. Es necesario desarrollar una capacitación en estandarización y analizar una mejor distribución física de las máquinas del taller, para mejorar el flujo de material de la empresa. El costo de una plancha industrial a vapor de 100 libras de presión, en promedio, es de \$1'600.000, junto con el salario de un operario que equivale a un salario mínimo y con los pagos correspondientes a sus prestaciones. Dicha inversión en maquinaria se recupera en un periodo no superior a tres meses, debido a que al incluir el costo del pago del nuevo operario se disminuye el margen de ganancias. Sin embargo, la labor social de incrementar en un operario la mano de obra y el incremento en los activos de la empresa incrementan la capacidad de endeudamiento para posteriores inversiones.

\section{CONCLUSIONES}

En este trabajo se ha realizado la representación y simulación de un proceso de confección de camisas masculinas en una pequeña empresa nacional. El objetivo principal fue identificar las falencias en los procesos actuales y proponer escenarios de solución orientados a incrementar la productividad de la compañía. El interés principal del proceso divulgativo es incentivar a empresas pequeñas el uso de herramientas de simulación de sistemas para optimizar sus procesos y ganar competitividad. La simulación del sistema actual permitió evidenciar demoras en el proceso productivo generadas por la utilización inapropiada de las máquinas existentes. Se simuló un escenario de solución del problema orientado a aumentar el recurso de plancha industrial en una unidad, a partir de un análisis de los procesos que generan represamientos en el sistema actual.

La evaluación del resultado ofrece una mejora superior al $10 \%$ en la productividad semanal de la empresa. Esto puede reflejar una mejor utilización de los recursos disponibles, lo cual conduce a mayores beneficios económicos relacionados con la rentabilidad de la empresa satisfaciendo la demanda semanal. No obstante, es necesario realizar un proceso de actualización de las máquinas que componen el proceso fileteado, ya que en este se observa la mayor parte del "cuello de botella" del sistema, lo que podría mejorarse con una inversión en tecnología.

En general, el análisis realizado permitió encontrar "cuellos de botella", estaciones de servicio clave y otros puntos en los cuales se 
pueda impactar con soluciones que ofrezcan un incremento en la productividad con el menor costo de forma similar a las reportadas en [28-29].

\section{BIBLIOGRAFÍA}

[1] Labarca, N. and Zulia, U. (2007.) "Consideraciones teóricas de la competitividad empresarial", Omnia, Vol. 13, No. 2, pp. 158-184.

[2] Gereffi, G. (1999). "International trade and industrial upgrading in the apparel commodity chain". J. Int. Econ., Vol. 48, No. 1, pp. 37-70, junio.

[3] De Toni, A. and Meneghetti, A. (2000). "The production planning process for a network of rms in the textile apparel industry," Int. J. Prod. ..., Vol. 65, 2.

[4] Davis, J., Eisenhardt, K. and Bingham, C. (2007). "Developing theory through simulation methods". Acad. Manag., Vol. 32, No. 2, pp. 480-499.

[5] Gilbert, N. (2007). "Computational social science: Agent-based social simulation". Comput. Soc. Sci. Agent-based Soc. Simul., pp. 115-134.

[6] Mourtzis, D., Doukas, M., Bernidaki, D. (2014). "Simulation in Manufacturing: Review and Challenges", Procedia CIRP, Volume 25, pp. 213-229, ISSN 22128271.

[7] Vélez, L., Rodríguez, E., Camacho, M. (2013). "Informe desempeño del sector textil de confecciones 2008-2012". Superintendencia de Sociedades. Bogotá.
[8] De Toni, A., Meneghetti, A. (2000). "The production planning process for a network of firms in the textileapparel industry". Int. J. Production Economics, Vol. 65, No. 1, pp. 17-32.

[9] Banu, Y., Arslan, M. (2008). "A simulation based experimental design to analyze factors affecting production flow time", Simulation Modelling Practice and Theory, Vol. 16, No. 1, pp. 278-293.

[10] Gómez, U., Gómez, O. (2013). "Modelo de simulación para el proceso de producción en empresa de confecciones textiles", Revista S\&T, Vol. 11, No. 24, pp. 73-89.

[11] Bevilacqua, M., Ciarapica, F., Crosta, A. Mazzuto, G. and Paciarotti. C. (2013). "Designing an efficient production system: A case study of a clothing company". International Journal of Engineering Bussines Management, Vol. 5, No. 36, pp. 1-8.

[12] Solano, M., Bravo, J. and Giraldo, J. (2012). "Metodología de mejoramiento en el desempeño de sistemas de producción. Aplicaciones en Pymes de la confección". Ingeniería y Competitividad, Vol. 14, No. 2, pp. 37-52.

[13] Guo, Z. X., Wong, W. K., Leung, S. Y. S., Fan, J. T. and Chan, S. F. (2006). "Mathematical model and genetic optimization for the job shop scheduling problem in a mixed- and multi-product assembly environment: $A$ case study based on the apparel industry". Comput. Ind. Eng., Vol. 50, No. 3, pp. 202219, julio.

[14] Robinson, S. (2004). Simulation: The Practice of Model Development and Use, 1st ed. Wiley. 
[15] Chang, Y. C., Chen, W. C., Yang, Y. N. and Chao, H. C. (2009). "A flexible web-based simulation game for production and logistics management courses". Simul. Model. Pract. Theory, Vol. 17, No. 7, pp. 1241-1253.

[16] Vits, J.(2002)."Performance improvement theory". Int. J. Prod. Econ., Vol. 77, No. 3, pp. 285-298, junio.

[17] Jahangirian, M., Eldabi, T., Naseer, A., Stergioulas, L. K. and Young, T. (2010). "Simulation in manufacturing and business: A review". Eur. J. Oper. Res., Vol. 203, No. 1, pp. 1-13.

[18] Smith, J. (2003). "Survey on the Use of Simulation for Manufacturing System Design and Operation". J. Manuf. Syst., Vol. 22, No. 2, pp. 157-171.

[19] Swets, R. J. and Drake, G. R. (2001). "The Arena product family: enterprise modeling solutions". Proceeding 2001 Winter Simul. Conf. (Cat. No. 01CH37304), Vol. 1.

[20] Statgraphics. (1988). "A Statistical Graphics Software System". Disasters, Vol. 12, No. 4, p. 18.

[21] Chapra. (2007). Métodos numéricos para ingenieros, McGraw-Hill Interamericana, 4th ed.

[22] Dyner, I., Peña, G. and Arango, S. (2008). Modelamiento para sistemas socioeconómicos y naturales, 1st ed. Medellín: Universidad Nacional de Colombia.
[23] Barlas, Y. and Kanar, K. (1997). "A Dynamic Pattern-oriented Test for Model Validation 1". Ind. Eng., No. 97.

[24] Samarghandi, H. and Eshghi, K. (2010). "An efficient tabu algorithm for the single row facility layout problem". Eur. J. Oper. Res., Vol. 205, No. 1, pp. 98-105, Aug.

[25] Carrillo, J., Gomis, R. (2003). "Los retos de las maquiladoras ante la pérdida de competitividad", Comercio Exterior, Vol. 53, No. 4, pp. 318-327, abril.

[26] Ngai, E., Peng, S., Alexander, P. y Moon, K. (2014). "Decision support and intelligent systems in the textile and apparel supply chain: An academic review of research articles", Expert Syst. Appl., Vol. 41, No. 1, pp. 81-91.

[27] Hincapié, S. and Saker, F. (2014). "Implementar un sistema de producción para la confección de camisas en Medellín para la marca Camisería Europea". Tesis de grado, Universidad Pontificia Bolivariana, Medellín.

[28] Degraeve, Z., Gochet, W. and Jans, R. (2002). "Alternative formulations for a layout problem in the fashion industry". Eur. J. Oper. Res., Vol. 143, No. 1, pp. 8093, Nov.

[29] Nascimento, D. B., Neiva de Figueiredo, J., Mayerle, S. F., Nascimento, P. R. and Casali, R. M. (2010). "A state-space solution search method for apparel industry spreading and cutting". Int. J. Prod. Econ., Vol. 128, No. 1, pp. 379-392, Nov. 